\title{
Discordant association of the CREBRF rs373863828 A allele with increased BMI and protection from type 2 diabetes in Māori and Pacific (Polynesian) people living in Aotearoa/New Zealand
}

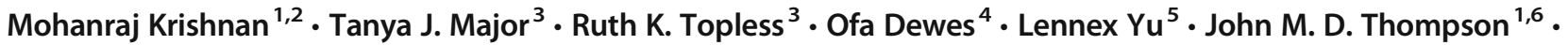 \\ Lesley McCowan ${ }^{1}$. Janak de Zoysa ${ }^{7}$. Lisa K. Stamp ${ }^{8}$. Nicola Dalbeth ${ }^{2}$. Jennie Harré Hindmarsh ${ }^{9}$. Nuku Rapana ${ }^{10}$. \\ Ranjan Deka ${ }^{11}$ - Winston W. H. Eng ${ }^{12}$. Daniel E. Weeks ${ }^{12,13}$ • Ryan L. Minster ${ }^{13}$. Stephen T. McGarvey ${ }^{14}$. \\ Satupa'itea Viali ${ }^{15}$. Take Naseri ${ }^{16}$. Muagututi'a Sefuiva Reupena ${ }^{17}$. Phillip Wilcox ${ }^{18}$ • David Grattan ${ }^{4,19}$. \\ Peter R. Shepherd ${ }^{2,4}$ • Andrew N. Shelling ${ }^{1} \cdot$ Rinki Murphy ${ }^{2,4}$ - Tony R. Merriman ${ }^{3,4}$
}

Received: 7 October 2017 / Accepted: 16 March 2018 / Published online: 2 May 2018

(C) Springer-Verlag GmbH Germany, part of Springer Nature 2018

\begin{abstract}
Aims/hypothesis The A (minor) allele of CREBRF rs373863828 has been associated with increased BMI and reduced risk of type 2 diabetes in the Samoan populations of Samoa and American Samoa. Our aim was to test rs373863828 for associations with BMI and the odds of type 2 diabetes, gout and chronic kidney disease (CKD) in Māori and Pacific (Polynesian) people living in Aotearoa/New Zealand.

Methods Linear and logistic regression models were used to analyse the association of the A allele of $C R E B R F$ rs373863828 with BMI, log-transformed BMI, waist circumference, type 2 diabetes, gout and CKD in 2286 adults. The primary analyses were adjusted for age, sex, the first four genome-wide principal components and (where appropriate) BMI, waist circumference and type 2 diabetes. The primary analysis was conducted in ancestrally defined groups and association effects were combined using meta-analysis.

Results For the A allele of rs373863828, the effect size was 0.038 (95\% CI $0.022,0.055, p=4.8 \times 10^{-6}$ ) for log-transformed BMI, with OR $0.59\left(95 \%\right.$ CI $\left.0.47,0.73, p=1.9 \times 10^{-6}\right)$ for type 2 diabetes. There was no evidence for an association of genotype with variance in BMI $(p=0.13)$, and nor was there evidence for associations with serum urate $\left(\beta=0.012 \mathrm{mmol} / 1, p_{\text {corrected }}=\right.$ 0.10 ), gout (OR 1.00, $p=0.98$ ) or CKD (OR 0.91, $p=0.59$ ).

Conclusions/interpretation Our results in New Zealand Polynesian adults replicate, with very similar effect sizes, the association of the A allele of rs373863828 with higher BMI but lower odds of type 2 diabetes among Samoan adults living in Samoa and American Samoa.
\end{abstract}

Keywords Association · BMI · CREBRF · Genetic · Māori · Obesity · Pacific · Polynesian · Type 2 diabetes

\begin{abstract}
Abbreviations
AIC Akaike's information criterion

CKD Chronic kidney disease
\end{abstract}

Mohanraj Krishnan and Tanya J. Major contributed equally as first authors. Rinki Murphy and Tony R. Merriman contributed equally as senior authors.

Electronic supplementary material The online version of this article (https://doi.org/10.1007/s00125-018-4623-1) contains peer-reviewed but unedited supplementary material, which is available to authorised users.

Tony R. Merriman

tony.merriman@otago.ac.nz

Extended author information available on the last page of the article
CREB3 cAMP-responsive element binding protein 3

CREBRF cAMP-responsive element binding protein

3 regulatory factor

MAF Minor allele frequency

\section{Introduction}

A missense variant (rs373863828, p.Arg457Gln) in CREBRF (encoding cAMP-responsive element binding protein 3 [CREB3] regulatory factor) has been associated with BMI in the Samoan and American Samoan populations [1]. The minor allele (c.1370A, p.457Gln) was associated with a $1.36 \mathrm{~kg} / \mathrm{m}^{2}$ greater BMI ( 4 kg in body weight at $1.7 \mathrm{~m}$ tall) 


\section{Research in context}

\section{What is already known about this subject?}

- The A (minor) allele of CREBRF rs373863828 (p.Arg457GIn), which is essentially unique to the Pacific population, is associated with increased BMl yet reduced risk of type 2 diabetes in Samoans living in Samoa and American Samoa, and with increased $\mathrm{BMI}$ in Tongans living in the Kingdom of Tonga

\section{What is the key question?}

- Does the A allele of rs373863828 have an association with increased BMI and reduced risk of type 2 diabetes in people of Māori and Pacific (Polynesian) ancestry living in Aotearoa/New Zealand?

\section{What are the new findings?}

- The A allele of rs373863828 was associated with increased BMI and reduced risk of type 2 diabetes in people of Māori and Pacific (Polynesian) ancestry living in Aotearoa/New Zealand

- There was, however, no association with serum urate levels, gout or chronic kidney disease

\section{How might this impact on clinical practice in the foreseeable future?}

- $\quad$ CREBRF participates in a newly discovered pathway linking type 2 diabetes and obesity, which may reveal new ways to manage diabetes

$[1,2]$. In a small sample from the Kingdom of Tonga $(n=171)$ the A (minor) allele was associated with an increase of $3.1 \mathrm{~kg} / \mathrm{m}^{2}$ in BMI [3]. The high A allele frequency of rs373863828 among Samoans in Samoa and American Samoa (minor allele frequency [MAF] 0.26) and in Tonga (MAF 0.15) $[1,3]$ is in contrast to an exceedingly rare frequency in other populations in the Genome Aggregation Database (http://gnomad.broadinstitute.org, accessed 1 October 2017; MAF $5.3 \times 10^{-5}$ in East Asian individuals, $3.3 \times 10^{-5}$ in South Asian individuals, $4.0 \times 10^{-5}$ in European individuals and absent in 7651 African individuals) [4] , and supports the hypothesis that rs373863828 is an important risk factor for obesity that is unique to Samoan and Tongan populations and possibly other Polynesian populations.

Unlike FTO-IRX3 and many other obesity risk variants in other populations, the BMI-increasing A allele of rs 373863828 has been associated with lower odds of type 2 diabetes (OR 0.59-0.74 after adjustment for BMI) [1]. This contrasts with the established observational positive association between type 2 diabetes and increased BMI. 'Favourable adiposity' genetic variants have recently been identified, which have been associated with a higher BMI but lower risk of type 2 diabetes, hypertension and coronary artery disease in European populations [5-7]. For example, in a study that used data from the UK Biobank, a genetic score of 11 such alleles was associated with a higher BMI $\left(+0.12 \mathrm{~kg} / \mathrm{m}^{2}\right)$ and higher body fat percentage $(+0.30 \%)$ in the top $50 \%$ of people carrying the most favourable adiposity alleles, compared to the bottom $50 \%$. However, for a given BMI, individuals carrying these alleles had reduced odds of type 2 diabetes (OR 0.84), hypertension (OR 0.94) and heart disease (OR 0.92) [8]. Such genetic variants are hypothesised to promote energy storage by expanding favourable subcutaneous adipose tissue depots, resulting in less accumulation of fat in non-adipose tissues and a consequent reduction in the risk of type 2 diabetes and other metabolic consequences of insulin resistance $[8,9]$. In contrast, aside from increasing BMI and decreasing both fasting glucose and the risk of type 2 diabetes, the $C R E B R F$ rs 373863828 A allele has not been associated with other features of insulin resistance such as hypertension, altered lipid levels or a higher HOMA-IR [1].

In Aotearoa/New Zealand, obesity and type 2 diabetes are both very prevalent in Māori and Pacific people [9] and are associated with other very prevalent metabolic-based conditions, in particular gout and chronic kidney disease (CKD) [10-12]. Moreover, increased BMI is a cause of increased urate levels [13], and diabetic kidney disease has been ascribed as the cause of renal failure in $69 \%$ of Polynesian people [14]. CREBRF rs373863828 has not previously been tested for an association with gout or CKD [1]. Polynesian populations include those from West Polynesia (Samoa, Tonga, Niue and Tokelau) and East Polynesia (Aotearoa/New Zealand Māori and Cook Island Māori). Given different pathogenic allele frequencies and different linkage disequilibrium structures between East and West Polynesian populations [15, 16], investigating CREBRF rs373863828 in other Polynesian population groups may provide further insights into its association with increasing BMI, yet apparent reduction in the risk of type 2 diabetes.

In this study, we tested the associations of CREBRF rs373863828 with BMI, waist circumference, type 2 diabetes, gout and CKD in people of Polynesian ancestry living in Aotearoa/New Zealand. In addition, based on the association of the FTO genotype with BMI variance in Europeans [17], we tested the association of rs 373863828 with variance in logtransformed BMI to detect possible underlying genetic and/or environmental influences on phenotypic variability. 


\section{Methods}

\section{Study population}

Individuals aged $\geq 16$ years, primarily from the Auckland, Waikato and Christchurch regions of Aotearoa/New Zealand, were recruited to the Genetics of Gout, Diabetes and Kidney Disease in Aotearoa New Zealand Study [18]. A separate Māori sample set from the rohe (area) of the Ngāti Porou iwi (tribe) of the Tairāwhiti (east coast of the North Island) region was also included in the Aotearoa/New Zealand Māori analysis. This sample set was recruited in collaboration with the Ngāti Porou Hauora (Health Service) Charitable Trust. A Pukapuka Island sample set was recruited in collaboration with the Pukapuka Community of New Zealand Inc. in Mangere, South Auckland.

Information obtained at recruitment included age, sex, height, weight and waist circumference, as measured by trained assessors. BMI was calculated by dividing an individual's weight by the square of their height in metres. Participants were also asked about the ancestry of each of their grandparents. Type 2 diabetes was ascertained by physician diagnosis, participant report or the use of glucose-lowering therapy. Blood biochemical measurements were performed at the Southern Community Laboratories (www.sclabs.co. nz). eGFR was derived from participants' serum creatinine, age and sex using the Chronic Kidney Disease Epidemiology Collaboration equation [19]. Stage 4 and 5 CKD were defined by an eGFR of $<30 \mathrm{ml} \mathrm{min}^{-1}[1.73 \mathrm{~m}]^{-2}$. Obesity was defined as a BMI of $>32 \mathrm{~kg} / \mathrm{m}^{2}$ [20]. Ethical approval for this study was given by the NZ Multi-Region Ethics Committee (MEC/05/10/130; MEC/10/09/092; MEC/ 11/04/036) and the Northern Y Region Health Research Ethics Committee (Ngāti Porou Hauora Charitable Trust study; NTY07/07/074). All participants provided written informed consent for the collection of samples and subsequent analysis.

Participants were separated into sample sets based on the self-reported Pacific nation of ancestry of their grandparents. Those participants who also reported nonPolynesian ancestry were grouped according to their Polynesian ancestry. This resulted in seven sample sets: Aotearoa/New Zealand Māori $(n=1296$, including 270 people from the Ngāti Porou Hauora Charitable Trust study), Cook Island Māori $(n=205)$, Samoan $(n=387)$, Tongan $(n=181)$, Niuean $(n=47)$, Pukapukan $(n=75)$ and an 'other' Polynesian group $(n=271)$, which included individuals of Tahitian $(n=3)$, Tokelauan $(n=6)$ and Tuvaluan $(n=5)$ ancestry, along with individuals who self-reported grandparental ancestry from more than one Pacific nation $(n=257)$. Pukapuka is part of the Cook Islands, but is geographically located within West Polynesia. These analysis groups were further refined based on clustering of genome-wide principal component vectors one to four (details of calculation below), resulting in the exclusion of 176 people (ESM Fig. 1) for one of three reasons: (1) a clear mismatch between self-reported and principal component-defined ancestry $(n=16$; potentially indicating an error in DNA or phenotype sample ID alignment during processing); (2) clustering outside of any of the principal component clusters $(n=26)$; and (3) individuals self-reported as Aotearoa/New Zealand Māori and Cook Island Māori who clustered within the European and between the European and Eastern Polynesian clusters $(n=135)$. The final groups used in all analyses totalled 2286 and were: Aotearoa/New Zealand Māori $(n=1154)$, Cook Island Māori $(n=197)$, Samoan $(n=378)$, Tongan $(n=175)$, Niuean $(n=47)$, Pukapukan $(n=70)$ and 'other' Polynesian $(n=265)$. Baseline characteristics for the final groupings are shown in Table 1.

\section{Whole-genome microarray analysis}

The Illumina Infinium CoreExome v24 BeadChip platform (Illumina, San Diego, CA, USA) was used to genotype participants for approximately 500,000 variants across the whole genome. Genotyping was performed at the University of Queensland (Centre for Clinical Genomics) for the Genetics of Gout, Diabetes and Kidney Disease in Aotearoa/New Zealand Study cohorts and at AgResearch (Invermay, Dunedin, New Zealand) for the Ngāti Porou Hauora Charitable Trust cohort. BeadChip genotyping batches were auto-clustered using GenomeStudio v2011.1 software (Illumina, San Diego, CA, USA). The Illumina GenomeStudio best practice guidelines and other qualitycontrol protocols were applied [21, 22]. The genotyping batches were then merged and relevant quality-control steps were repeated for the full dataset.

\section{Determination of principal components}

Whole-genome principal component analysis vectors were calculated using a subset of 2858 ancestry-informative markers (as identified by Illumina) extracted from the CoreExome whole-genome genotypes. The SmartPCA (EIGENSOFT v6.0.1; www.hsph.harvard.edu/alkes-price/ software/) [23] program was used, with an output of ten eigenvectors, no outlier removal and no population size limit. Individuals of non-Polynesian ancestry were included, and the first four vectors were plotted against each other to view the clustering of ancestral groupings (Asian, European, Eastern Polynesian and Western Polynesian) (ESM Fig. 1). The first four vectors, which explained $97.1 \%$ of the proportion of the variability within the sample sets, were included as covariates in the linear and logistic regression analyses to account for population stratification and cryptic relatedness. 
Table 1 Baseline characteristics

\begin{tabular}{|c|c|c|c|c|c|c|c|}
\hline Characteristic & NZ Māori & Cook Island Māori & Samoan & Tongan & Niuean & Pukapukan & $\begin{array}{l}\text { 'Other' } \\
\text { Polynesian }\end{array}$ \\
\hline$n$ & 1154 & 197 & 378 & 175 & 47 & 70 & 265 \\
\hline Male & $666(57.7)$ & $117(59.4)$ & $280(74.1)$ & $131(74.9)$ & $36(76.6)$ & $33(47.1)$ & $162(61.1)$ \\
\hline Age (years) & $52.25 \pm 14.66$ & $52.16 \pm 14.28$ & $45.25 \pm 13.50$ & $43.61 \pm 14.67$ & $48.28 \pm 12.72$ & $44.14 \pm 17.28$ & $42.38 \pm 15.41$ \\
\hline Weight (kg) & $96.97 \pm 23.93$ & $100.73 \pm 25.28$ & $106.90 \pm 23.05$ & $108.06 \pm 21.61$ & $96.88 \pm 18.78$ & $95.16 \pm 20.85$ & $104.63 \pm 28.35$ \\
\hline Height $(\mathrm{cm})$ & $169.57 \pm 9.55$ & $169.03 \pm 9.99$ & $173.39 \pm 9.84$ & $174.18 \pm 8.53$ & $171.73 \pm 8.16$ & $165.56 \pm 9.34$ & $171.59 \pm 8.30$ \\
\hline BMI $\left(\mathrm{kg} / \mathrm{m}^{2}\right)$ & $33.79 \pm 7.96$ & $35.37 \pm 8.56$ & $35.63 \pm 7.84$ & $35.55 \pm 6.88$ & $32.77 \pm 5.09$ & $34.60 \pm 6.61$ & $35.43 \pm 8.42$ \\
\hline $\begin{array}{l}\text { Waist } \\
\text { circumference }(\mathrm{cm})\end{array}$ & $108.04 \pm 18.24$ & $110.75 \pm 15.85$ & $111.80 \pm 16.28$ & $112.53 \pm 14.72$ & $106.06 \pm 12.78$ & $108.20 \pm 16.07$ & $110.06 \pm 18.81$ \\
\hline Serum urate $(\mathrm{mmol} / \mathrm{l})^{\mathrm{a}}$ & $0.363 \pm 0.11$ & $0.406 \pm 0.096$ & $0.395 \pm 0.13$ & $0.385 \pm 0.13$ & $0.413 \pm 0.11$ & $0.405 \pm 0.092$ & $0.387 \pm 0.11$ \\
\hline $\begin{array}{l}\text { eGFR }\left(\mathrm{ml} \mathrm{min}^{-1}\right. \\
\left.[1.73 \mathrm{~m}]^{-2}\right)\end{array}$ & $71.23 \pm 28.42$ & $69.36 \pm 28.70$ & $72.49 \pm 27.31$ & $73.00 \pm 31.76$ & $70.94 \pm 26.68$ & $79.79 \pm 22.23$ & $76.25 \pm 28.25$ \\
\hline Triacylglycerol $(\mathrm{mmol} / \mathrm{l})^{\mathrm{b}}$ & $2.25 \pm 1.53$ & $2.37 \pm 1.96$ & $2.13 \pm 1.40$ & $2.21 \pm 1.43$ & $2.52 \pm 1.92$ & $2.09 \pm 1.40$ & $2.10 \pm 1.43$ \\
\hline HDL-cholesterol $(\mathrm{mmol} / \mathrm{l})^{\mathrm{b}}$ & $1.16 \pm 0.36$ & $1.17 \pm 0.39$ & $1.10 \pm 0.34$ & $1.09 \pm 0.37$ & $1.10 \pm 0.33$ & $1.21 \pm 0.23$ & $1.13 \pm 0.37$ \\
\hline LDL-cholesterol $(\mathrm{mmol} / \mathrm{l})^{\mathrm{b}}$ & $2.80 \pm 1.01$ & $2.88 \pm 0.99$ & $2.87 \pm 1.05$ & $2.82 \pm 1.06$ & $3.03 \pm 1.21$ & $2.71 \pm 1.05$ & $2.76 \pm 0.93$ \\
\hline Type 2 diabetes & $301(27.3)$ & $63(33.0)$ & $83(22.6)$ & $49(29.0)$ & $10(21.3)$ & $16(22.9)$ & $61(23.4)$ \\
\hline Gout & $456(43.4)$ & $96(51.3)$ & $207(57.0)$ & $92(53.2)$ & $21(50.0)$ & $11(16.4)$ & $100(40.7)$ \\
\hline CKD & $115(11.9)$ & $23(13.9)$ & $31(9.5)$ & $23(14.6)$ & $4(11.1)$ & $0(0.0)$ & $24(10.1)$ \\
\hline \multicolumn{8}{|l|}{ rs373863828 genotype } \\
\hline $\mathrm{G} / \mathrm{G}$ & $772(67.4)$ & $127(65.1)$ & $212(56.8)$ & $120(68.6)$ & $39(83.0)$ & $42(60.0)$ & $168(64.1)$ \\
\hline $\mathrm{G} / \mathrm{A}$ & $348(30.4)$ & $60(30.8)$ & $146(39.1)$ & $47(26.9)$ & 7 (14.9) & $22(31.4)$ & $77(29.4)$ \\
\hline $\mathrm{A} / \mathrm{A}$ & $25(2.2)$ & $8(4.1)$ & $15(4.0)$ & $8(4.6)$ & $1(2.1)$ & $6(8.6)$ & $17(6.5)$ \\
\hline HWE $p$ value & 0.049 & 0.79 & 0.098 & 0.23 & 0.34 & 0.22 & 0.052 \\
\hline \multicolumn{8}{|l|}{ Allele } \\
\hline A & $398(17.4)$ & $76(19.5)$ & $176(23.6)$ & $63(18.0)$ & $9(9.6)$ & $34(24.3)$ & $111(21.2)$ \\
\hline
\end{tabular}

Data are mean \pm SD or $n(\%)$, unless otherwise stated

The proportions with type 2 diabetes, gout, rs373863828 genotype and A allele are calculated from individuals without missing data

${ }^{a}$ Serum urate concentrations are reported from individuals not taking urate-lowering therapy

${ }^{\mathrm{b}}$ Data were unavailable for lipid-lowering medications

HWE, Hardy-Weinberg equilibrium; NZ, Aotearoa/New Zealand

\section{CREBRF rs373863828 genotyping}

rs373863828 was directly genotyped because this variant was not present on the CoreExome platform and we were unable to impute the region owing to the unavailability of Māori and Pacific reference haplotypes. A customdesigned TaqMan probe-set (Applied Biosystems, Foster City, CA, USA) was created for rs373863828 using a custom Python script (snp_design; DOI: https://doi.org/ 10.5281/zenodo.56250) to annotate the human genome build 37 reference sequence (ftp://ftp.ensembl.org/pub/ grch37; accessed 1 August 2016) with rs373863828 and any surrounding SNPs (obtained from the NCBI dbSNP build 147 common SNP list; ftp://ftp.ncbi.nlm.nih.gov/ snp). Forward primer: CAAGAGAGGATGCTGAGACC AT; reverse primer: ACCATGATGTAAGCCATTTT TCTGATACA; probe 1 (VIC): TGAGTGGA ACCGAGATAC probe 2 (FAM): AGTGGAAC CAAGATAC. Genotyping was performed using the
LightCycler 480 Real-Time Polymerase Chain Reaction System (Roche Applied Science, Indianapolis, IN, USA) in 384 well plates. There was a $99 \%$ successful genotyping call rate. Re-genotyping of $25 \%$ of the sample set demonstrated $100 \%$ concordance.

\section{Association testing}

Analyses were performed using the R v3.3.2 statistical software (within RStudio v0.99.902; www.rstudio.com). A multivariable linear regression model was used to test for associations between the rs373863828 minor allele (c. $1370 \mathrm{~A}$, p. $457 \mathrm{Gln})$ and the continuous variables logtransformed BMI (a Box-Cox normality plot for the BMI data yielded $\lambda=-0.39$ ), untransformed BMI, waist circumference and serum urate, with the $\beta$ coefficient representing the estimated effect of each copy of the rs373863828 A allele. For binary outcomes (obesity, type 2 diabetes, gout and $\mathrm{CKD}$ ), a multivariable binomial 
logistic regression model was used in a similar manner, with the allelic OR representing the estimated effect of each copy of the A allele. All analyses were adjusted for sex, age, the first four whole-genome principal component vectors and BMI, type 2 diabetes or waist circumference, where appropriate. Analyses were also performed in groups stratified by type 2 diabetes status. Each Polynesian population sample set was analysed separately, and the effects were combined using an inverse-varianceweighted fixed-effect meta-analysis. Heterogeneity among sample sets was assessed during the meta-analysis using Cochran's heterogeneity $(\mathrm{Q})$ statistic, with a randomeffects analysis used when there was evidence of heterogeneity $(p<0.05)$. For the BMI, waist circumference and type 2 diabetes association analyses, $p<0.05$ was set as the significance value, given the prior probability of detecting associations [1]. For the other outcomes $(n=4$; serum urate, gout, CKD and variance in BMI), $p<$ 0.0125 was set as the significance value, accounting for the fact that none of these outcomes had previously been tested for associations with rs373863828 [1].

The association analyses for the major outcomes (BMI, $\log _{e}$-transformed BMI and type 2 diabetes) were repeated using two different approaches to account for relatedness within the analysis group. First, a kinship coefficient matrix was calculated in PLINK v1.9 [24] using 40,156 independent autosomal markers in the pooled dataset. Participant pairs with a kinship coefficient $(\hat{\pi}) \geq 0.125$ (equivalent to first cousins or closer) were identified and one individual was excluded (at random) to create a maximal set of unrelated individuals $(n=1665)$ for analysis. Multivariate linear (or logistic) regression analyses testing the CREBRF rs373863828 association were performed in this group, adjusting for sex, age, the first four principal component vectors, membership of the seven previously described ancestral groups and BMI, type 2 diabetes or waist circumference, where appropriate. Second, for BMI, a linear mixed-model regression analysis, which included the kinship coefficient matrix calculated above to adjust for relatedness, was performed in the entire analysis group (not excluding related individuals) using GenABEL (www. rdocumentation.org/packages/GenABEL/versions/1.8-0) [25, $26]$ and the same adjusting variables as above.

Models of inheritance were investigated by formulating the genotype predictor in the linear and logistic regression models (adjusting for age, sex and principal components $1-4)$ in different ways: additive model $(0,1,2)$; dominant model $(0,1,1)$; or recessive model $(0,0,1)$. For each model the coded genotypes were treated as a continuous variable, producing a single effect estimate ( $\beta$ or OR). A model-selection tool (Akaike's information criterion [AIC] [27]) was used to select the most likely model. Smaller AIC values indicate better models, but where the difference was $<2$ the simplest model was chosen.

\section{Power}

Based on previously reported estimates [1] and $\alpha=0.05$, the power to detect an effect size of $1.36 \mathrm{~kg} / \mathrm{m}^{2}$ per A allele was $>80 \%$ in the combined Māori and Pacific Island sample set for a MAF of $\geq 0.15$ (ESM Fig. 2) The power to detect a moderate protective effect for type 2 diabetes (OR 0.59 ) of the minor allele was $>90 \%$ for a MAF of $\geq 0.10$ (ESM Fig. 2). Power calculations for gout and CKD as outcomes with $\alpha=0.0125$ (to account for serum urate, gout, CKD and variance in BMI, not previously tested [1]) showed that the power was adequate only to detect effect sizes of OR $\geq 1.75$ for gout and CKD and $\geq 0.032 \mathrm{mmol} / \mathrm{l}$ for serum urate (ESM Fig. 2).

\section{Testing for an association of rs373863828 with variance in log-transformed BMI}

The association of a genetic variant with variance in phenotype can detect a locus interacting in a non-additive way without prior knowledge of the interacting factor (environmental, intrinsic, genetic). Testing for an association of rs 373863828 with variance in log-transformed BMI was performed as previously described [28]. The variable used as a measure of variance was produced from residuals obtained from cohortspecific analyses regressing age, age ${ }^{2}$ and age by sex. An independent ranked inverse normal transformation of absolute residuals generated $Z$ scores, with squared $Z$ scores $\left(Z^{2}\right)$ being the variance variable. To account for the influence of rs373863828 mean effect on the variance, the mean logtransformed BMI (per genotype) was subtracted from the log-transformed BMI of each participant and the $Z$ scores were recalculated. Linear models associating CREBRF genotype with both the unadjusted and mean-effect adjusted variance $Z$ scores were used. This analysis was also performed on the Samoan rs373863828 genotype data [1], with age, age ${ }^{2}$, age by sex and polity as the adjusting variables in the $Z$ score calculation steps. The Aotearoa/New Zealand sample sets and the two Samoan sample sets [1] were combined using an inverse-variance weighted fixed-effect metaanalysis [28].

\section{Results}

\section{Prevalence of rs373863828}

The allele and genotype frequencies of rs373863828 in each Polynesian sample set are presented in Table 1 and Fig. 1. There was no evidence of deviation from Hardy-Weinberg equilibrium in the various sample sets (Table $1 ; p$ for all datasets was $\geq 0.049$ ). The relative frequencies of the minor (c.1370A) allele differed among the Polynesian groups, with the Samoan (MAF 0.236) and Pukapukan (MAF 0.243) 

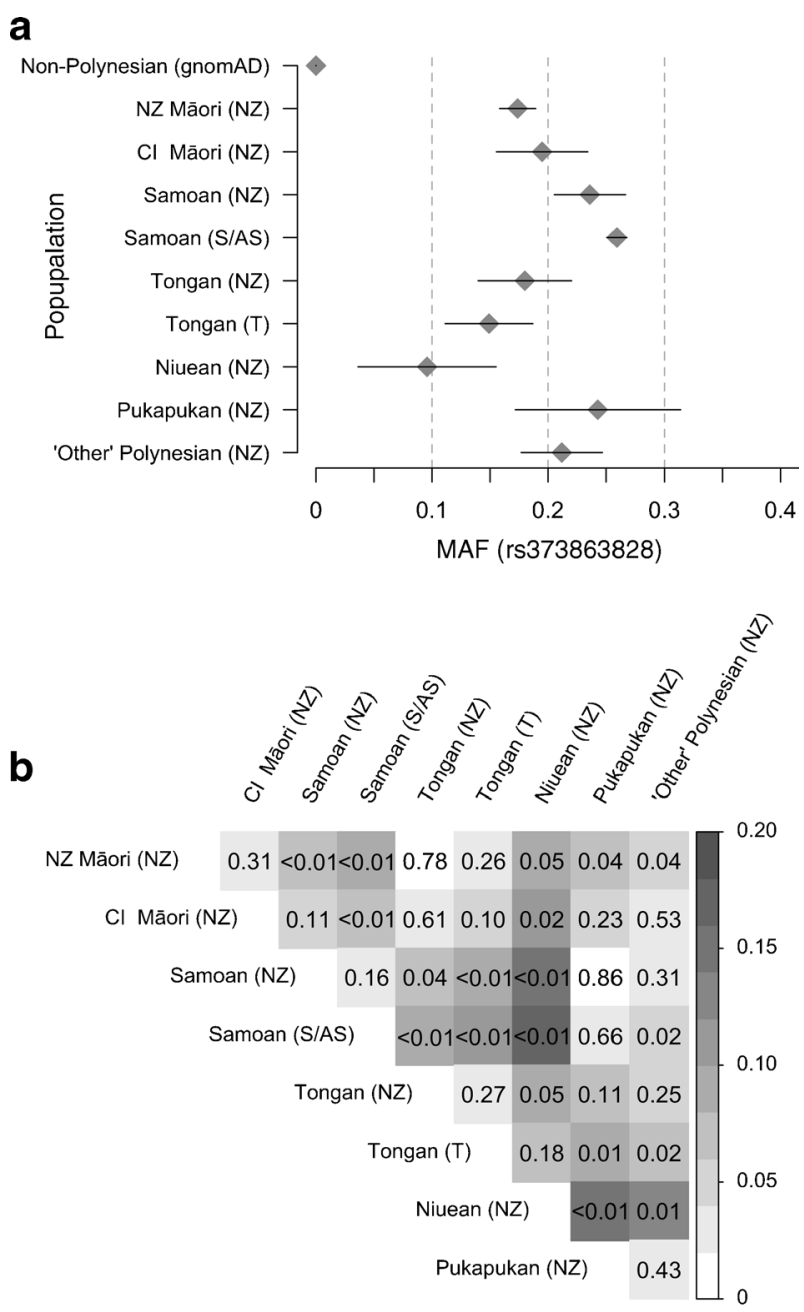

Allele frequency differences (rs373863828)

Fig. 1 Frequencies of the rs373863828 A allele in various Māori and Pacific ancestral groups (a) and a comparison of allele frequencies among the Māori and Pacific ancestral groups (b). The $p$ values shown in (b) are derived from a difference in the proportions parametric $Z$ test. Squares are shaded according to the difference in allele frequency (key in right-hand vertical bar). AS, American Samoa; NZ, Aotearoa/New Zealand; S, Samoa; T, Kingdom of Tonga. 'Non-Polynesian' includes all populations in the Genome Aggregation Database (gnomAD; http://gnomad. broadinstitute.org)

groups exhibiting the highest frequencies and the Niuean (MAF 0.096) group exhibiting the lowest frequency (Table 1).

\section{Association analysis of rs373863828 with adiposity measures}

A fixed-effect meta-analysis of the Polynesian samples showed a significant association of rs373863828 with logtransformed BMI $\left(\beta=0.038, p=4.8 \times 10^{-6}\right)$, with no evidence of heterogeneity among the sample sets $(p=0.19)$ (Table 2, Fig. 2; ESM Table 1). Association analysis with untransformed BMI revealed similar results (Table 2, Fig. 2; ESM Table 2). Analysis of the pooled dataset removing related individuals yielded very similar effect sizes ( $\beta=0.037$ [95\% CI $0.017,0.057$ ], $p=2.3 \times 10^{-4}$ for logtransformed BMI and $\beta=1.38 \mathrm{~kg} / \mathrm{m}^{2}$ [95\% CI $\left.0.66,2.09\right]$, $p=1.6 \times 10^{-4}$ for untransformed BMI). Analysis adjusting for relatedness using GenABEL also yielded very similar effect sizes $\left(\beta=0.038\right.$ [95\% CI 0.021, 0.054], $p=1.2 \times 10^{-5}$ for log-transformed BMI and $\beta=1.34 \mathrm{~kg} / \mathrm{m}^{2}$ [95\% CI 0.73 , $1.94], p=1.6 \times 10^{-5}$ for untransformed BMI). The proportion of variance explained by rs 373863828 in the pooled dataset was $1.1 \%$. One copy of the minor allele was sufficient to confer the effect (Table $2 ; \beta=1.80$ for the heterozygote group and $\beta=1.49$ for the A allele homozygote group compared with major allele homozygotes in untransformed BMI analysis). This was supported by AIC analysis, where the dominant model had a difference of 3.3 less than the additive model. In the combined group there was an association with higher odds of obesity (Table 2; OR 1.33, $p=8 \times 10^{-4}$ for BMI $>32 \mathrm{~kg} / \mathrm{m}^{2}$ and OR 1.54, $p=1 \times 10^{-5}$ for BMI $>40 \mathrm{~kg} / \mathrm{m}^{2}$ ). There was a significant association with untransformed BMI in both the type 2 diabetes-positive and -negative groups $\left(\beta=1.62 \mathrm{~kg} / \mathrm{m}^{2}, p=2.4 \times 10^{-3}\right.$ and $\beta=1.21 \mathrm{~kg} / \mathrm{m}^{2}$, $p=5.0 \times 10^{-4}$, respectively, with no evidence of heterogeneity in effect size $\left.\left[p_{\mathrm{Het}}=0.48\right]\right)$, indicating no evidence of a type 2 diabetes-specific effect on BMI. There was no indication of sex-specific effects (Table 2, Fig. 3) and a sex-byrs373863828 interaction analysis of the pooled sample set showed no evidence of an interaction with BMI $(p=0.67)$.

A fixed-effect meta-analysis showed evidence of an association between rs373863828 and increased waist circumference in the full Polynesian sample set $(\beta=2.98 \mathrm{~cm}$ per minor allele, $p=1.3 \times 10^{-5}$ ), with no evidence of heterogeneity among sample sets $(p=0.089)$ (Fig. 2; ESM Table 3). Adjustment of the waist circumference analysis for BMI abrogated the association with waist circumference (Table $2 ; \beta$ decreased from 2.98 to $0.66, p=0.092$ ).

\section{Association analysis of rs373863828 with type 2 diabetes}

A fixed-effect meta-analysis of the various sample sets revealed a significant association with reduced odds of type 2 diabetes (OR $0.65, p=3.4 \times 10^{-5}$ ) that was strengthened after adjustment for BMI (OR 0.59, $p=1.9 \times 10^{-6}$ ), with no evidence of heterogeneity among sample sets $(p=0.089)$ (Table 2, Fig. 4; ESM Table 4). Association analysis of the pooled dataset with related individuals removed yielded very similar effect sizes (OR 0.56 [95\% CI 0.43, 0.72], $p=7.9 \times$ $\left.10^{-6}\right)$. Similar to the observation for BMI, one copy of the minor allele appeared to be sufficient to confer the effect (Table 2: OR 0.55 for the heterozygote group compared with the major allele homozygote group). The AIC analysis showed the dominant model to be similar to the additive model $(0.21$ less). Therefore, the mode of inheritance for type 2 
Table 2 Association of rs373863828 with weight measures, type 2 diabetes, gout and CKD in the full Polynesian sample set meta-analysis

\begin{tabular}{|c|c|c|c|c|}
\hline Traits & Covariables & $n$ & $\begin{array}{l}\beta(95 \% \mathrm{CI}) \text { continuous or OR } \\
(95 \% \mathrm{CI}) \text { dichotomous }\end{array}$ & $p$ value \\
\hline \multicolumn{5}{|l|}{ Continuous traits } \\
\hline Log-transformed BMI, all & 4 PCA vectors, sex, age, T2D & 2125 & $0.038(0.022,0.055)$ & $4.8 \times 10^{-6}$ \\
\hline Male & 4 PCA vectors, age, T2D & 1335 & $0.042(0.023,0.062)$ & $2.4 \times 10^{-5}$ \\
\hline Female & 4 PCA vectors, age, T2D & 790 & $0.032(0.0014,0.062)$ & 0.040 \\
\hline Untransformed BMI $\left(\mathrm{kg} / \mathrm{m}^{2}\right)$, all & 4 PCA vectors, sex, age, T2D & 2125 & $1.38(0.78,1.98)$ & $7.3 \times 10^{-6}$ \\
\hline Untransformed BMI $\left(\mathrm{kg} / \mathrm{m}^{2}\right)$, all & 4 PCA vectors, sex, age, T2D, waist & 1890 & $0.16(-0.18,0.51)$ & 0.35 \\
\hline Male & 4 PCA vectors, age, T2D & 1335 & $1.51(0.78,2.23)$ & $4.4 \times 10^{-5}$ \\
\hline Female & 4 PCA vectors, age, T2D & 790 & $1.17(0.089,2.26)$ & 0.034 \\
\hline T2D positive & 4 PCA vectors, sex, age & 557 & $1.62(0.57,2.67)$ & $2.4 \times 10^{-3}$ \\
\hline $\mathrm{T} 2 \mathrm{D}$ negative & 4 PCA vectors, sex, age & 1568 & $1.21(0.53,1.90)$ & $5.0 \times 10^{-4}$ \\
\hline Major allele homozygote & - & 1381 & - & - \\
\hline Heterozygote & 4 PCA vectors, sex, age, T2D & 670 & $1.80(1.08,2.53)$ & $9.7 \times 10^{-7}$ \\
\hline Minor allele homozygote & 4 PCA vectors, sex, age, T2D & 74 & $1.49(-0.32,3.30)$ & 0.11 \\
\hline Waist circumference $(\mathrm{cm})$, all & 4 PCA vectors, sex, age, T2D & 1904 & $2.98(1.64,4.33)$ & $1.4 \times 10^{-5}$ \\
\hline Waist circumference $(\mathrm{cm})$, all & 4 PCA vectors, sex, age, T2D, BMI & 1890 & $0.66(-0.11,1.42)$ & 0.092 \\
\hline Male & 4 PCA vectors, age, T2D & 1221 & $2.81(1.23,4.40)$ & $5.010^{-4}$ \\
\hline Female & 4 PCA vectors, age, T2D & 683 & $3.29(0.80,5.78)$ & $9.7 \times 10^{-3}$ \\
\hline Urate $(\mathrm{mmol} / \mathrm{l})^{\mathrm{a}}$ & 4 PCA vectors, sex, age & 1237 & $0.015(0.005,0.026)$ & $5.0 \times 10^{-3}$ \\
\hline Urate $(\mathrm{mmol} / \mathrm{l})^{\mathrm{a}}$ & 4 PCA vectors, sex, age, BMI & 1199 & $0.012(0.0014,0.022)$ & 0.026 \\
\hline Urate $(\mathrm{mmol} / \mathrm{l})$, T2D positive ${ }^{\mathrm{a}}$ & 4 PCA vectors, sex, age & 266 & $0.0038(-0.030,0.038)$ & 0.83 \\
\hline Urate $(\mathrm{mmol} / \mathrm{l}), \mathrm{T} 2 \mathrm{D}$ negative $^{\mathrm{a}}$ & 4 PCA vectors, sex, age & 944 & $0.012(0.0014,0.022)$ & 0.026 \\
\hline Urate $(\mathrm{mmol} / \mathrm{l}), \mathrm{T} 2 \mathrm{D}$ positive ${ }^{\mathrm{a}}$ & 4 PCA vectors, sex, age, BMI & 255 & $-0.018(-0.049,0.014)$ & 0.28 \\
\hline Urate $(\mathrm{mmol} / \mathrm{l}), \mathrm{T} 2 \mathrm{D}$ negative $^{\mathrm{a}}$ & 4 PCA vectors, sex, age, BMI & 917 & $0.011(0.0003,0.021)$ & 0.043 \\
\hline \multicolumn{5}{|l|}{ Dichotomous traits } \\
\hline Obesity $\left(\mathrm{BMI}>32 \mathrm{~kg} / \mathrm{m}^{2}\right)$ & 4 PCA vectors, sex, age, T2D & 2125 & $1.33(1.13,1.58)$ & $8 \times 10^{-4}$ \\
\hline Obesity $\left(\mathrm{BMI}>40 \mathrm{~kg} / \mathrm{m}^{2}\right)$ & 4 PCA vectors, sex, age, T2D & 2125 & $1.54(1.27,1.87)$ & $1.0 \times 10^{-5}$ \\
\hline $\mathrm{T} 2 \mathrm{D}$, all & 4 PCA vectors, sex, age & 2190 & $0.65(0.53,0.80)$ & $3.4 \times 10^{-5}$ \\
\hline $\mathrm{T} 2 \mathrm{D}$, all & 4 PCA vectors, sex, age, BMI & 2125 & $0.59(0.48,0.73)$ & $1.9 \times 10^{-6}$ \\
\hline Male & 4 PCA vectors, age, BMI & 1335 & $0.54(0.41,0.72)$ & $1.5 \times 10^{-5}$ \\
\hline Female & 4 PCA vectors, age, BMI & 779 & $0.66(0.46,0.95)$ & 0.026 \\
\hline Major allele homozygote & - & 1381 & - & - \\
\hline Heterozygote & 4 PCA vectors, sex, age, BMI & 670 & $0.55(0.43,0.71)$ & $3.2 \times 10^{-6}$ \\
\hline Minor allele homozygote & 4 PCA vectors, sex, age, BMI & 74 & $0.72(0.34,1.53)$ & 0.39 \\
\hline Gout & 4 PCA vectors, sex, age & 2114 & $1.10(0.91,1.32)$ & 0.34 \\
\hline Gout & 4 PCA vectors, sex, age, BMI, T2D & 2009 & $1.00(0.81,1.22)$ & 0.98 \\
\hline Gout, T2D positive ${ }^{\mathrm{b}}$ & 4 PCA vectors, sex, age & 498 & $1.03(0.71,1.52)$ & 0.86 \\
\hline Gout, T2D negative & 4 PCA vectors, sex, age & 1549 & $1.08(0.86,1.36)$ & 0.50 \\
\hline Gout, T2D positive ${ }^{\mathrm{b}}$ & 4 PCA vectors, sex, age, BMI & 480 & $0.89(0.59,1.33)$ & 0.57 \\
\hline Gout, T2D negative & 4 PCA vectors, sex, age, BMI & 1510 & $0.99(0.77,1.26)$ & 0.91 \\
\hline $\mathrm{CKD}$ & 4 PCA vectors, sex, age & 1849 & $0.72(0.53,0.97)$ & 0.030 \\
\hline CKD & 4 PCA vectors, sex, age, BMI & 1795 & $0.72(0.53,0.99)$ & 0.045 \\
\hline CKD & 4 PCA vectors, sex, age, T2D & 1810 & $0.86(0.63,1.15)$ & 0.36 \\
\hline CKD & 4 PCA vectors, sex, age, BMI, T2D & 1756 & $0.91(0.65,1.28)$ & 0.59 \\
\hline CKD, T2D positive & 4 PCA vectors, sex, age & 488 & $0.71(0.46,1.09)$ & 0.11 \\
\hline CKD, T2D negative & 4 PCA vectors, sex, age & 1322 & $1.12(0.67,1.87)$ & 0.66 \\
\hline CKD, T2D positive & 4 PCA vectors, sex, age, BMI & 470 & $0.80(0.50,1.27)$ & 0.34 \\
\hline CKD, T2D negative & 4 PCA vectors, sex, age, BMI & 1286 & $1.12(0.65,1.93)$ & 0.69 \\
\hline
\end{tabular}

${ }^{\text {a }}$ Analysis excluded individuals taking urate-lowering medication

${ }^{\mathrm{b}}$ Analysis excluded individuals of Niuean and Pukapukan ancestry due to small sample size

PCA, principal component analysis; T2D, type 2 diabetes

diabetes was concluded to be more consistent with an additive model.

We combined all available data. A fixed-effect meta-analysis that included the data presented here and data from the previously published Samoan [1] and Tongan [3] studies yielded $\beta=1.43 \mathrm{~kg} / \mathrm{m}^{2}(95 \%$ CI $1.17,1.68), p=3.8 \times 10^{-28}$ for BMI and OR 0.62 (95\% CI 0.55, 0.70), $p=1.7 \times 10^{-14}$ for type 2 diabetes, with no evidence of heterogeneity.

\section{Association analysis of rs373863828 with serum urate, gout and CKD}

We tested for associations with serum urate (Table 2; ESM Fig. 3). Unadjusted, there was evidence of an association of the $\mathrm{A}$ allele with higher levels $(\beta=0.015 \mathrm{mmol} / 1, p=0.005$, $\left.p_{\text {corrected }}=0.020\right)$; however, this effect was mitigated after adjusting for BMI $\left(\beta=0.012 \mathrm{mmol} / 1, p=0.026, P_{\text {corrected }}\right.$ 


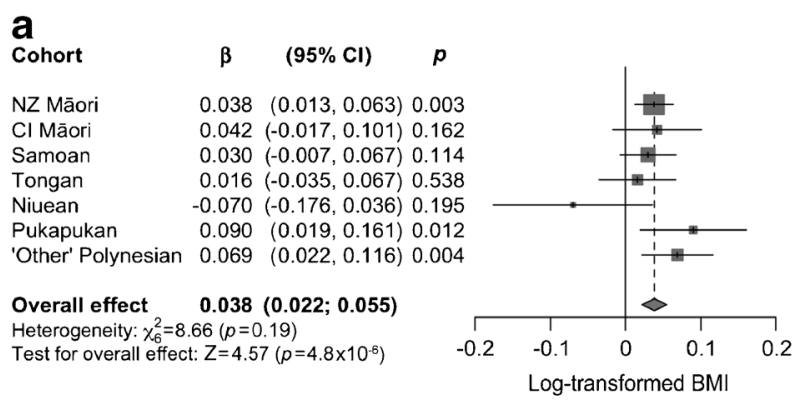

b

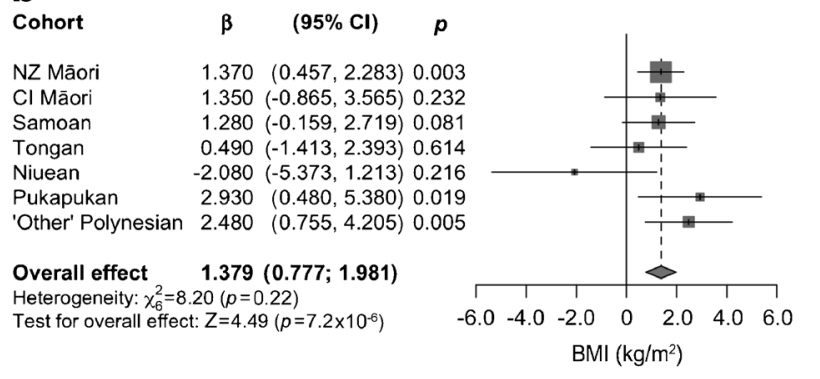

C

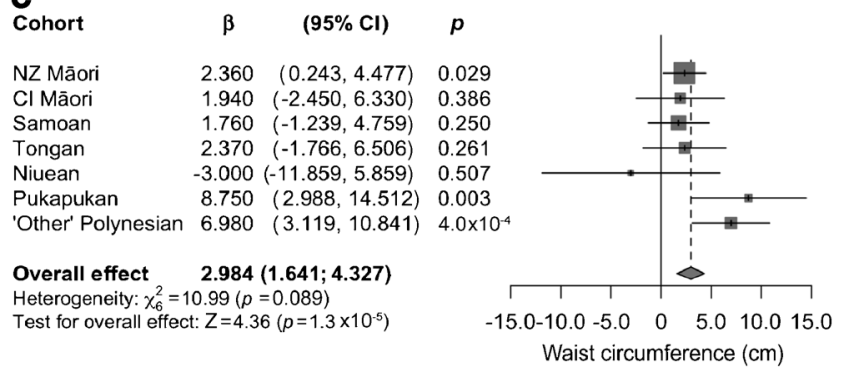

Fig. 2 Forest plot of a fixed-effect meta-analysis for the association of rs373863828 with log-transformed BMI (a), untransformed BMI (b) and waist circumference (c). Associations were adjusted for age, sex, the first four principal component analysis vectors and type 2 diabetes. CI Māori, Cook Island Māori; NZ, Aotearoa/New Zealand

$=0.10)$. Similarly there was no evidence of an association with gout (OR 1.00, $p=0.98$ ) (Table 2; ESM Table 5, ESM Fig. 3). There was no statistically significant $(p<0.0125) \mathrm{ev}-$ idence of an association with CKD either before (OR 0.72 , $p=0.030, p_{\text {corrected }}=0.12$ ) or after adjustment for type 2 diabetes and BMI (OR 0.91, $p=0.59$ ) (Table 2; ESM Table 6, ESM Fig. 3). Finally, analysis in type 2 diabetespositive and -negative groups yielded evidence of an association only for serum urate in the type 2 diabetes-negative group (Table 2). However there was no evidence of a difference in effect size between the type 2 diabetes-positive and -negative groups for serum urate $(p>0.13)$.

\section{Association analysis of rs373863828 with variance in phenotype}

The rs373863828 variant was not significantly associated with log-transformed BMI variance at the CREBRF locus for any of the Aotearoa/New Zealand sample sets or for the two previously published Samoan cohorts [1]. Fixed-effect meta-
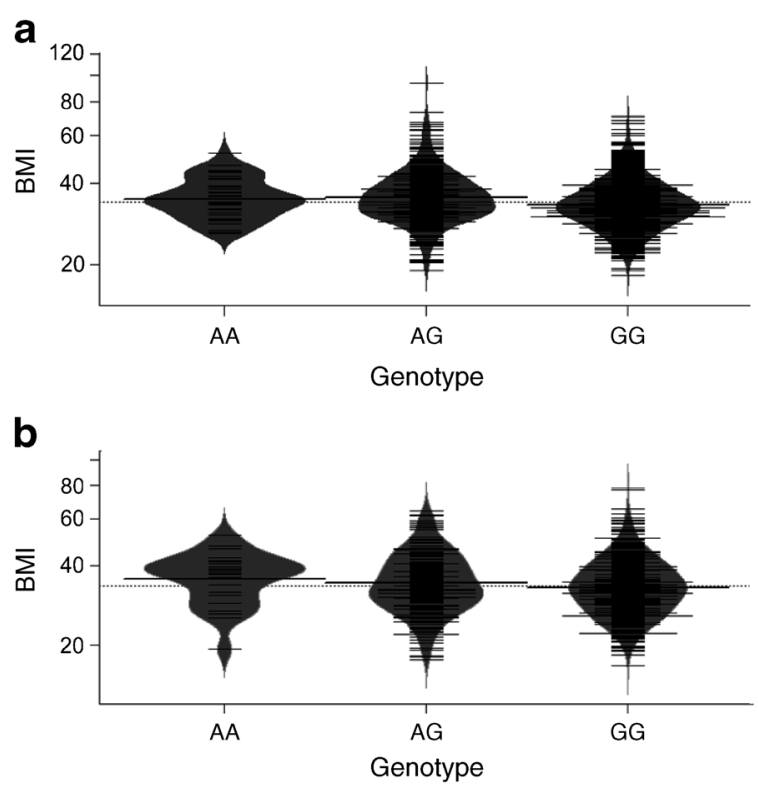

Fig. 3 Beanplots of $\log _{e}$ BMI vs the rs373863828 genotype in men (a) and women (b). Solid lines show the average for each group and dotted lines show the overall average. Plots were generated using the $\mathrm{R}$ beanplot package [32]

analysis of the Aotearoa/New Zealand $(n=2282), 1990 \mathrm{~s}$ Samoan $(n=1020)$ and discovery Samoan $(n=1876)$ cohorts showed no evidence of an association of rs373863828 with variance in log-transformed BMI in either the unadjusted $(\beta=$ $0.065, p=0.05)$ or adjusted models $(\beta=0.050, p=0.13)$ (ESM Table 7, ESM Fig. 4).

\section{Discussion}

In this study, we confirmed the association of $C R E B R F$ rs 373863828 with a higher BMI $\left(1.38 \mathrm{~kg} / \mathrm{m}^{2}\right.$ per minor allele) and larger waist circumference $(2.98 \mathrm{~cm})$, but lower risk of type 2 diabetes (OR 0.59) in adults of Polynesian (Māori and Pacific) ancestry living in Aotearoa/New Zealand. These results are very similar to those from Samoans living in Samoa and American Samoa, where each copy of the minor allele was associated with a $1.36 \mathrm{~kg} / \mathrm{m}^{2}$ increase in BMI and an

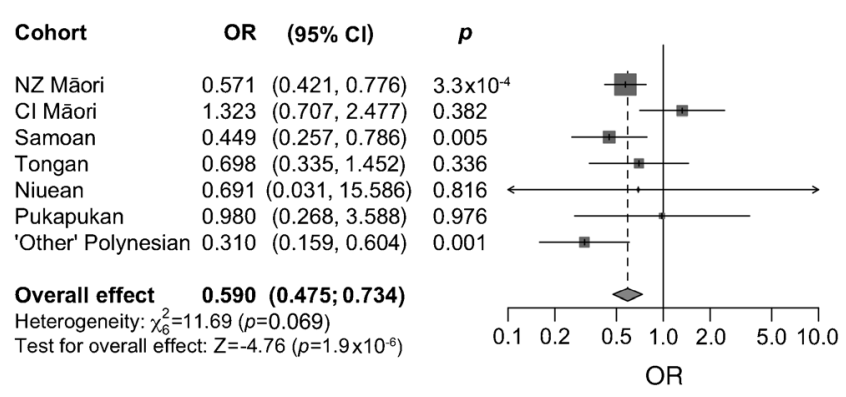

Fig. 4 Forest plot of a fixed-effect meta-analysis for the association of rs373863828 with type 2 diabetes. Associations were adjusted for age, sex, the first four principal component analysis vectors and BMI. CI Māori, Cook Island Māori; NZ, Aotearoa/New Zealand 
OR for type 2 diabetes of 0.59 [1]. The per cent variation in BMI explained was also similar $(1.9 \%$ in the Samoan discovery sample and $1.1 \%$ in the Samoan replication sample, vs $1.1 \%$ in our Aotearoa/New Zealand sample), suggesting that the main effect of the rs373863828 A allele is relatively impervious to environment. Consistent also with the Samoan data [1], adjustment for BMI strengthened the association with type 2 diabetes in the Aotearoa/New Zealand sample set (OR decreased from 0.65 to 0.59 [Table 2]; Samoan discovery OR decreased from 0.64 to 0.59 [1]; Samoan replication OR decreased from 0.83 to 0.74 [1]). However, adjustment for BMI diminished the association with waist circumference (Table 2), indicating that the waist circumference association was driven by overall body mass distribution rather than central adiposity.

Precisely how these genetic epidemiological findings relate to the actual CREBRF-mediated molecular pathogenesis of obesity and type 2 diabetes is unclear in the absence of detailed knowledge of the molecular pathways involving CREBRF and in the absence of genetic association data with detailed body-composition measures as outcomes. Most population genetic variants associated with generalised obesity are also associated with insulin resistance, hypertension, dyslipidaemia and type 2 diabetes, compatible with the degree of adiposity. However, some genetic variants are associated with a higher BMI and percentage body fat, but lower odds of type 2 diabetes along with lower insulin resistance, hypertension, circulating triacylglycerols and LDL-cholesterol levels. These variants are known as 'favourable adiposity' variants [8] due to higher subcutaneous-to-visceral adipose tissue that suggests preferential fat storage away from visceral organs. The association of the A allele of rs373863828 with a higher BMI but reduced odds of type 2 diabetes is not entirely compatible with 'favourable adiposity' due to the lack of an association with hypertension or lipids [1]. Consistent with the reduced odds of type 2 diabetes, there was a weak association of the A allele with increased insulin sensitivity by HOMA-IR in the Samoan and American Samoan populations [1]; HOMA-IR data were not available for the current study. Cellular bioenergetics models have shown that the rs373863828 A allele promotes lipid and triacylglycerol storage in adipocytes at a reduced energy cost, suggesting that the metabolic activity of CREBRF in fat is important [1]. Detailed clinical studies are required to clarify whether visceral and subcutaneous body fat storage depots are altered among carriers of this variant.

It is notable that, from what is currently understood about the physiological role of CREBRF, it has no obvious role in regulating appetite, which is seen at FTO-IRX3 and other loci regulating BMI in Europeans. However, $C R E B R F$ is widely expressed (www.gtexportal.org), including throughout the brain, and CREBRF is known to regulate the CREB3/ Luman protein, which is localised to the endoplasmic reticulum and plays an important role in axonal regeneration [29]. Interestingly, the CREB3/Luman protein was identified through its association with herpes simplex virus-related host cell factor 1, which has led to the hypothesis that CREB3/ Luman may play a role in emergence of the virus from latency [30]. It will be important to explore the relationship between $C R E B R F$ expression in the hypothalamic nuclei and the role of CREB3/Luman in intra-axonal translation and retrograde trafficking to promote neuronal survival in response to viral stimuli, and how this might relate to BMI and type 2 diabetes.

A recent study reported an association of the CREBRF rs373863828 A allele with higher BMI in a pooled multiethnic sample of 4572 New Zealand children of Māori, Pacific, European and Asian ethnicity [31]. Given that the association analysis was pooled and population-specific association analyses were not reported, we are unable to directly compare our data with that study. The A allele was reported at a prevalence of 0.015 in European children and 0.011 in Asian children [31], in contrast to the very low prevalence reported in the Genome Aggregation Database (MAF $5.3 \times 10^{-5}$ in East Asian individuals, $3.3 \times 10^{-5}$ in South Asian individuals and $4.0 \times 10^{-5}$ in European individuals).

Our study has confirmed that the presence of each additional CREBRF rs373863828 A allele is associated with increased BMI yet reduced odds of type 2 diabetes in adults of Māori and Pacific (Polynesian) ancestry living in Aotearoa/ New Zealand. While the prevalence of both obesity and type 2 diabetes is increased among New Zealand Māori and those of Pacific ethnicity [9] compared with New Zealand Europeans, our study confirms that Pacific-specific genetic variation underpins some of the interindividual heterogeneity observed in the discordant manifestations of obesity and type 2 diabetes. This study supports the need to conduct comprehensive genephenotype studies in populations currently under-represented in genomic studies, among which different genetically segregating pathways linking obesity and type 2 diabetes clearly exist. Such studies are not only important for these populations per se, but are also important in illuminating the molecular biology of the pathogenesis of metabolic disease in the wider human population and have the potential to lead to novel clinical interventions.

Acknowledgements The authors sincerely thank the participants for generously donating their time and information to this study. The authors would like to thank J. Drake (Department of Rheumatology, Canterbury District Health Board, Christchurch, New Zealand), J. de Kwant, R. Laurence, C. Franklin and M. House (all Department of Medicine, University of Auckland, Auckland, New Zealand), N. Aupouri, R. Akuhata and C. Ford (all of Ngāti Porou Hauora Charitable Trust, Te Puia Springs, New Zealand) and G. Sexton (Counties Manukau District Health Board, Auckland, New Zealand) for recruitment.

Data availability The dataset generated during the current study is not publicly available owing to consent restrictions, but can be requested from the corresponding author under an appropriate arrangement. 
Funding The Health Research Council of New Zealand (grant no. 08/ 075, 10/548, 11/1075, 14/527) and the Maurice Wilkins Centre funded the New Zealand component of this study, and the National Institutes of Health funded the Samoa and American Samoa components (grant no. R01-HL093093 and R01-HL133040).

Duality of interest The authors declare that there is no duality of interest associated with this manuscript.

Contribution statement MK, TJM, PRS, RM and TRM contributed to the design of the study. OD, LM, JdZ, LKS, ND, JHH, NR, TN, MSR, RD, STM and SV contributed to data collection, and RKT, LY, JMDT, WWHE, DEW, RLM, PW, DG and ANS contributed to data analysis and interpretation. MK, RM and TRM drafted the manuscript and all of the other authors reviewed it. The manuscript was approved by all authors. TRM is the guarantor of this work.

\section{References}

1. Minster RL, Hawley NL, Su CT et al (2016) A thrifty variant in CREBRF strongly influences body mass index in Samoans. Nat Genet 48:1049-1054

2. Loos RJ (2016) CREBRF variant increases obesity risk and protects against diabetes in Samoans. Nat Genet 48:976-978

3. Naka I, Furusawa T, Kimura R et al (2017) A missense variant, rs373863828-A (p.Arg457Gln), of CREBRF and body mass index in Oceanic populations. J Hum Genet 62:847-849

4. Lek M, Karczewski KJ, Minikel EV et al (2016) Analysis of protein-coding genetic variation in 60,706 humans. Nature 536: 285-291

5. Kilpelainen TO, Zillikens MC, Stancakova A et al (2011) Genetic variation near IRS1 associates with reduced adiposity and an impaired metabolic profile. Nat Genet 43:753-760

6. Yaghootkar H, Scott RA, White CC et al (2014) Genetic evidence for a normal-weight "metabolically obese" phenotype linking insulin resistance, hypertension, coronary artery disease, and type 2 diabetes. Diabetes 63:4369-4377

7. Scott RA, Fall T, Pasko D et al (2014) Common genetic variants highlight the role of insulin resistance and body fat distribution in type 2 diabetes, independent of obesity. Diabetes 63:4378-4387

8. Yaghootkar H, Lotta LA, Tyrrell J et al (2016) Genetic evidence for a link between favorable adiposity and lower risk of type 2 diabetes, hypertension, and heart disease. Diabetes 65:2448-2460

9. Virtue S, Vidal-Puig A (2010) Adipose tissue expandability, lipotoxicity and the metabolic syndrome - an allostatic perspective. Biochim Biophys Acta 1801:338-349

10. Winnard D, Wright C, Jackson G et al (2012) Gout, diabetes and cardiovascular disease in the Aotearoa New Zealand adult population: co-prevalence and implications for clinical practice. N Z Med J 126:53-64

11. Winnard D, Wright C, Taylor WJ et al (2012) National prevalence of gout derived from administrative health data in Aotearoa New Zealand. Rheumatology (Oxford) 51:901-909

12. Hollis-Moffatt JE, Phipps-Green AJ, Chapman B et al (2012) The renal urate transporter SLC17A1 locus: confirmation of association with gout. Arthritis Res Ther 14:R92
13. Robinson PC, Choi HK, Do R, Merriman TR (2016) Insight into rheumatological cause and effect through the use of Mendelian randomization. Nat Rev Rheumatol 12:486-496

14. Australia and New Zealand Dialysis \& Transplant Registry (2015) Available from www.anzdata.org.au/v1/report 2015.html. Accessed 26 Sept 2017

15. Friedlaender JS, Friedlaender FR, Reed FA et al (2008) The genetic structure of Pacific islanders. PLoS Genet 4:e19

16. Terrell JE (2010) Social network analysis of the genetic structure of Pacific islanders. Ann Hum Genet 74:211-232

17. Yang J, Loos RJ, Powell JE et al (2012) FTO genotype is associated with phenotypic variability of body mass index. Nature 490:267272

18. Phipps-Green AJ, Merriman ME, Topless R et al (2016) Twentyeight loci that influence serum urate levels: analysis of association with gout. Ann Rheum Dis 75:124-130

19. Levey AS, Stevens LA, Schmid CH et al (2009) A new equation to estimate glomerular filtration rate. Ann Intern Med 150:604-612

20. Swinburn BA, Ley SJ, Carmichael HE, Plank LD (1999) Body size and composition in Polynesians. Int J Obes Relat Metab Disord 23: $1178-1183$

21. Guo Y, He J, Zhao S et al (2014) Illumina human exome genotyping array clustering and quality control. Nat Protoc 9:2643-2662

22. Illumina (2014) Infinium genotyping data analysis: a guide for analyzing infinium genotyping data using the GenomeStudio genotyping module. Available from https://www.illumina.com/ Documents/products/technotes/technote_infinium_genotyping data_analysis.pdf

23. Price AL, Patterson NJ, Plenge RM, Weinblatt ME, Shadick NA, Reich D (2006) Principal components analysis corrects for stratification in genome-wide association studies. Nat Genet 38:904-909

24. Purcell S, Neale B, Todd-Brown K et al (2007) PLINK: a toolset for whole-genome association and population-based linkage analysis. Am J Hum Genet 81:559-575

25. Aulchenko YS, Ripke S, Isaacs A, van Dujin CM (2007) GenABEL: an R library for genome-wide association analysis. Bioinformatics 23:1294-1296

26. Chen WM, Abecasis GR (2007) Family-based association tests for genome-wide association scans. Am J Hum Genet 81:913-926

27. Akaike H (1974) A new look at the statistical model identification. IEEE Trans Autom Control 19:716-723

28. Topless RK, Flynn TJ, Cadzow M et al (2015) Association of SLC2A9 genotype with phenotypic variability of serum urate in pre-menopausal women. Front Genet 6:313

29. Ying Z, Misra V, Verge VM (2014) Sensing nerve injury at the axonal ER: activated Luman/CREB3 serves as a novel axonally synthesized retrograde regeneration signal. Proc Natl Acad Sci U S A 111:16142-16147

30. Liang G, Audas TE, Li Y et al (2006) Luman/CREB3 induces transcription of the endoplasmic reticulum (ER) stress response protein Herp through an ER stress response element. Mol Cell Biol 26:7999-8010

31. Berry S, Walker C, Ly K et al (2017) Widespread prevalence of a CREBRF variant amongst Māori and Pacific children is associated with weight and height in early childhood. Int J Obes. https://doi. org/10.1038/ijo.2017.230

32. Kampstra P (2008) Beanplot: a boxplot alternative for visual comparison of distributors. J Stat Softw 28:1-9 


\section{Affiliations}

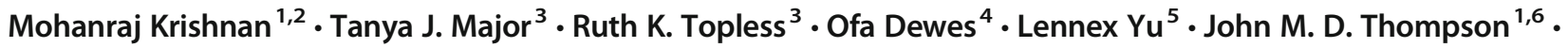
Lesley McCowan ${ }^{1}$. Janak de Zoysa ${ }^{7}$. Lisa K. Stamp ${ }^{8}$. Nicola Dalbeth ${ }^{2}$. Jennie Harré Hindmarsh ${ }^{9}$. Nuku Rapana ${ }^{10}$. Ranjan Deka ${ }^{11}$. Winston W. H. Eng ${ }^{12}$. Daniel E. Weeks ${ }^{12,13} \cdot$ Ryan L. Minster ${ }^{13}$. Stephen T. McGarvey ${ }^{14}$. Satupa'itea Viali ${ }^{15} \cdot$ Take Naseri $^{16} \cdot$ Muagututi'a Sefuiva Reupena $^{17}$. Phillip Wilcox ${ }^{18}$. David Grattan ${ }^{4,19}$. Peter R. Shepherd ${ }^{2,4}$ • Andrew N. Shelling ${ }^{1}$ - Rinki Murphy ${ }^{2,4}$ • Tony R. Merriman ${ }^{3,4}$

1 Department of Obstetrics and Gynaecology, University of Auckland, Auckland, New Zealand

2 Department of Medicine, Faculty of Medical and Health Sciences, University of Auckland University of Auckland, Auckland, New Zealand

3 Department of Biochemistry, University of Otago, 710 Cumberland Street, Dunedin 9054, New Zealand

4 Maurice Wilkins Centre for Molecular Biodiscovery, Auckland, New Zealand

5 Department of Anatomy and Medical Imaging, University of Auckland, Auckland, New Zealand

6 Department of Paediatrics, Child and Youth Health, University of Auckland, Auckland, New Zealand

7 Renal Services, Waitemata District Health Board, Auckland, New Zealand

8 Department of Medicine, University of Otago Christchurch, Christchurch, New Zealand

9 Ngāti Porou Hauora Charitable Trust, Te Puia Springs, Tairāwhiti East Coast, New Zealand
10 Pukapuka Community of New Zealand Inc., Mangere, Auckland, New Zealand

11 Department of Environmental Health, University of Cincinnati College of Medicine, Cincinnati, OH, USA

12 Department of Biostatistics, Graduate School of Public Health, University of Pittsburgh, Pittsburgh, PA, USA

13 Department of Human Genetics, Graduate School of Public Health, University of Pittsburgh, Pittsburgh, PA, USA

14 International Health Institute, Department of Epidemiology, Brown University School of Public Health, and Department of Anthropology, Brown University, Providence, RI, USA

15 Samoa National Health Service, Apia, Samoa

16 Ministry of Health, Government of Samoa, Apia, Samoa

17 Bureau of Statistics, Government of Samoa, Apia, Samoa

18 Department of Mathematics and Statistics, University of Otago, Dunedin, New Zealand

19 Department of Anatomy, University of Otago, Dunedin, New Zealand 